\title{
PETITIO PRINCIPII, AD IGNORANTIAM Y FUNDAMENTACIÓN DEL CONOCIMIENTO
}

\author{
JaVier Vilanova ARIaS \\ Departamento de Lógica \\ Facultad de Filosofía \\ Universidad Complutense de Madrid \\ vilanova@filos.ucm.es
}

RESUMEN: Se examina el problema de la circularidad argumental en la fundamentación del conocimiento desde la teoría de la argumentación. Los argumentos dirigidos a probar que sabemos (argumentos fundamentadores) son sospechosos de dos falacias: petición de principio y apelación a la ignorancia. Se examinan las definiciones clásicas de ambas falacias y se dictamina que bajo tales definiciones los argumentos fundamentadores serían falaces. A continuación se proporcionan nuevas definiciones de ambas falacias, más acordes con el trabajo reciente en teoría de la argumentación y se sostiene que, bajo estas definiciones, no todos los argumentos fundamentadores son falaces.

PALABRAS CLAVE: argumentación, circularidad argumental, elusión del problema, justificación epistémica

SUMMARY: In this paper I examine the problem of circularity in the justification of knowledge from the point of view of argumentation theory. I introduce the expression founding argument for the arguments we use to try to prove that we know, and explain the classical account of the two fallacies that may be committed: ad ignorantiam and petitio principii. A new definition of both fallacies, based on recent work in the theory of argumentation, is used to show that not every founding argument is fallacious.

KEY WORDS: argumentation, argument circularity, begging the question, epistemic justification

\section{Fundamentación del conocimiento}

En las páginas que siguen me ocuparé de una pequeña parte de ese gran problema que llamamos fundamentación del conocimiento. Y el aspecto que me preocupa no es otro que las suspicacias que desde el punto de vista lógico (o, en una perspectiva más amplia, desde el punto de vista de la teoría de la argumentación) despiertan los argumentos con los que intentamos averiguar si sabemos.

Para poder ir al grano cuanto antes, expondré el problema a través del siguiente argumento, que reconstruye el que Moore 1940 ofrece como prueba de que sus sentidos son una buena fuente para obtener conocimientos en torno al mundo "fuera de su cabeza". El argumento de Moore posee una cantidad innumerable de premisas, cada una de las cuales describe una ocasión en la que sus sentidos le informaron 
de algún hecho que fue corroborado por otras vías. Así, por ejemplo, en $t_{1}$ sus ojos le informaron de que algo sólido se aproximaba a su cara y más tarde sintió un impacto en su rostro, o en $t_{2}$ sus ojos le informaron de que había una silla al otro extremo del pasillo y a continuación su amigo Juan le corroboró tal hecho. Podemos esquematizar el argumento (que Moore califica como "inductivo" o "analógico") de la siguiente manera (es importante que tengamos en cuenta que la evidencia perceptiva para $\mathrm{M}_{n}$ no es la misma que la expresada por $\mathrm{M}_{n}$ bis):

\section{ARGUMENTO MOORE}

$\left(\mathrm{M}_{1}\right) \quad$ En $t_{1}$ mis sentidos me informaron de que $p_{1}$.

( $\mathrm{M}_{1}$ bis) Es el caso que $p_{1}$.

$\left(\mathrm{M}_{2}\right) \quad$ En $t_{2}$ mis sentidos me informaron de que $p_{2}$.

( $\mathrm{M}_{2}$ bis) Es el caso que $p_{2}$.

$\cdots$

$\left(\mathrm{M}_{n}\right) \quad$ En $t_{n}$ mis sentidos me informaron de que $p_{n}$.

( $\mathrm{M}_{n}$ bis) Es el caso que $p_{n}$.

(M)

Mis sentidos son fiables (es decir, aquello de lo que me informan mis sentidos es el caso).

Inmediatamente detectamos de dónde vienen las suspicacias con MOORE, y es que al menos parte de las razones que Moore posee para creer cada una de las premisas $\mathrm{M}_{n}$ bis es de origen perceptivo, y por lo tanto sólo es genuina su justificación si sus sentidos son fiables. Así que, prima facie, parece que Moore ya debería saber M antes de seguir este argumento, y por lo tanto el argumento no parece ser una vía adecuada para que Moore pruebe que sus sentidos son fiables. En este trabajo defenderé que, a pesar de las apariencias, no hay nada malo en el argumento MOORE. Por el contrario, el siguiente argumento, que reproduce el que aparece en Cohen 2002, es en mi opinión (como en la de Cohen) un intento fallido de probar la conclusión:

\section{ARGUMENTO COHEN}

$\left(C_{1}\right)$ Hay un objeto rojo encima de la mesa.

$\left(\mathrm{C}_{2}\right)$ Si hay un objeto rojo encima de la mesa, entonces en este momento mis sentidos proporcionan información fiable en torno al mundo externo. 
$\left(\mathrm{C}_{3}\right)$ Por lo tanto, mis sentidos proporcionan información fiable sobre el mundo externo en este momento.

La premisa $\left(\mathrm{C}_{1}\right)$ se justifica a partir de la experiencia perceptiva que estoy teniendo en este momento de que hay un objeto rojo encima de la mesa. La premisa $\left(\mathrm{C}_{2}\right)$ se obtiene a través del razonamiento que, a partir del hecho de que hay un objeto rojo encima de la mesa y del dato de que yo he llegado a saber tal cosa a través del uso de los sentidos, concluye que al menos en esta ocasión mis sentidos han sido una fuente verídica de conocimiento sobre el mundo externo. ${ }^{1}$

A esta característica de un argumento de "suponer" o utilizar la conclusión de alguna manera (ya que, como veremos, hay varias formas en que esto puede suceder) antes de haber llegado a ella se le conoce como circularidad argumental o, más brevemente, como circularidad. En el siguiente apartado efectuaremos un análisis más pormenorizado de la circularidad argumental y las dificultades ligadas a ella, pero ahora quisiera hacer unos comentarios generales sobre el tipo de tarea que se pretende llevar a cabo con argumentos como los anteriores. Como ya hemos dicho antes, ese tipo de tarea queda reunida bajo el rótulo de fundamentación del conocimiento, que consiste en la obtención de justificaciones de que sabemos. Explicaré esto un poco mejor.

Llamaré procedimientos o acciones epistémicas a aquellas acciones que llevamos a cabo con el fin de obtener nuevas creencias y conocimientos. Como resultado de una acción epistémica obtenemos una nueva información, un nuevo elemento de juicio a tener en cuenta a la hora de formarnos una creencia. Llamaré a tales informaciones evidencias. Se han postulado distintas fuentes de evidencias, que según el hombre o la época han sido aceptadas o no (los sentidos, la intuición racional, el testimonio, el sentido común, la revelación divina, etc.). Cuando las evidencias que han dado lugar a la creencia en $p$ son, objetivamente, una buena razón para creer que $p$, diré que son una justificación, o una buena justificación para creer $p$.

Por otro lado, una acción epistémica casi nunca constituye un acto aislado. Habitualmente se integra dentro de un sistema en el que

${ }^{1}$ El lector habrá percibido una notable diferencia entre ambos argumentos: MOORE es un argumento inductivo, mientras que COHEN es un argumento deductivo. Me apresuraré a advertir que, en mi opinión, el carácter deductivo o inductivo del argumento no afecta en absoluto al fenómeno de la circularidad. En la sección 8 de este trabajo fundamentaré esta afirmación y explicaré mejor este punto. 
han sido fijados (al menos idealmente) los procedimientos a seguir a la hora de recoger evidencias, y es en el marco de la metodología global del programa de investigación y de los datos ya obtenidos que la acción epistémica puede servir de justificación para una creencia. Debemos tener en cuenta, pues, procedimientos epistémicos dentro de los cuales se encontrarán las acciones epistémicas concretas que llevemos a cabo. Un procedimiento epistémico es un tipo de acción epistémico; por ejemplo, aplicar un modus ponens a proposiciones que recogen creencias ya justificadas, o sumergir una sustancia en agua regia, y comprobar si se disuelve o no. Un sistema epistémico será un conjunto de procedimientos epistémicos. La forma más sencilla de entender un sistema epistémico es como un conjunto de reglas, a las que llamaré reglas epistémicas, que al ser aplicadas nos proporcionan justificaciones para nuestras evidencias. Una regla epistémica típica sería: lleva a cabo la acción epistémica $X$, si obtienes el dato $Y$, entonces estás justificado para creer $Z$. Por último, llamaré proyecto epistémico a cualquier sistema epistémico enriquecido con una serie de objetivos o preguntas más una serie de evidencias previas y quizás algunos supuestos (podemos identificar un proyecto epistémico con lo que más tarde llamaré un contexto epistémico, así que dejaré la explicación para más tarde).

En una aproximación un tanto grosera pero efectiva, podemos definir la tarea de fundamentación del conocimiento como la tarea de obtención de justificaciones suficientes para nuestras afirmaciones de que sabemos, o, lo que es lo mismo, justificaciones de que las evidencias que respaldan nuestras creencias son buenas justificaciones. A los argumentos que, como MOORE o COHEN, pretenden obtener tales justificaciones los denominaré argumentos fundamentadores. Un par de observaciones aclararán mejor estas nociones.

En primer lugar, que nuestras evidencias son buenas justificaciones está directamente relacionado con la "calidad", la "fiabilidad" si se prefiere, de nuestras acciones epistémicas. Dicha fiabilidad depende en parte, claro está, de la situación concreta en que ha sido llevada a cabo. Especialmente se vería dañada si concurren circunstancias excepcionales que hacen imposible aplicar una regla epistémica que normalmente funciona bien. Pero además y sobre todo depende de la fiabilidad del procedimiento epistémico instanciado en la acción y la regla epistémica correspondiente. Y dado que, como hemos dicho, casi siempre (y al menos en los casos interesantes) la evidencia de una acción epistémica sólo puede entenderse como justificación en el marco de un conjunto estructurado de reglas epistémicas, la tarea de fundamentación del conocimiento no es más que la defensa de 
nuestros sistemas epistémicos. Una clase de sistemas epistémicos son los programas de investigación que estructuran la actividad científica, y los momentos en que el problema de la fundamentación científica se maximiza coinciden, evidentemente, con las crisis científicas. Pero también debemos tomar en consideración sistemas y procedimientos más modestos, que en algún momento pueden suscitar preguntas sobre su credibilidad. El recetario tradicional para prevenir el tiempo que viene, el uso de enciclopedias (o internet) para resolver dudas geográficas, o el recurso a la encuesta para conocer el estado de opinión de una población, pueden todos ellos embarcarnos en tareas de fundamentación del conocimiento.

En segundo lugar, entiendo que el objetivo de fundamentar el conocimiento es activado por una duda genuina cuya solución, positiva o negativa, no sólo afecta a nuestras pretensiones de conocimiento sino que tiene consecuencias prácticas para nuestros proyectos epistémicos, ya que nos puede llevar a abandonarlos o emprender serias reformas si el resultado es negativo, o a afianzarnos en ellos si es positivo. En este sentido, los casos interesantes, y los únicos que me ocuparán aquí, son aquellos en los que hay una posibilidad real de que la respuesta a la pregunta sobre si sabemos sea negativa. Es importante que reparemos en que pudiera haber ocurrido que Moore o cualquier otra persona, al recopilar sus experiencias perceptivas pasadas, detectara contradicciones, huecos y otros problemas que le llevaran a desconfiar del uso de sus sentidos y replantearse la manera en que obtiene conocimiento a través de ellos. Por poner un ejemplo - un tanto trivial pero bastante gráfico-, todos los que somos miopes en algún momento hemos seguido un razonamiento similar al de Moore, pero con conclusiones negativas en cuanto - eso sí, exclusivamente - al sentido de la vista. Pero lo mismo les ocurre, en todo el mundo, a personas que sufren graves trastornos de la percepción como agnosias o afasias mnésicas, o incluso desórdenes psicológicos tales como en el célebre caso del matemático John Nash.

En tercer lugar, no es necesario que un argumento fundamentador pruebe por sí solo de manera definitiva e irrefutable su conclusión para que sea pertinente. Basta con que añada algo de plausibilidad, o que aumente el grado de confirmación de la conclusión. Este rasgo se sigue de un hecho general señalado por la teoría de la argumentación, según la cual en las situaciones reales nunca o casi nunca se consigue probar un enunciado mediante un solo argumento, sino que son necesarios una serie de argumentos convergentes que van eliminando alternativas y sumando plausibilidad a la proposición que se quiere probar. No es necesario, pues, que un argumento como 
MOORE decida de una vez y para siempre la cuestión de la fiabilidad del conocimiento perceptivo, algo ya de por sí difícil si se tiene en cuenta que el argumento no es de naturaleza deductiva. Basta con que, en el momento en que se formula, sea una buena razón (entre y junto a otras) para confiar en nuestros sentidos, incluso aunque la aparición de evidencias posteriores nos hagan retractarnos de nuestra postura de confianza.

En buena medida, si insisto en estas consideraciones previas es con el objeto de aclarar que, tal y como yo lo entiendo, el problema de la fundamentación del conocimiento no es un mero pasatiempo filosófico, ni las dudas escépticas simples retos intelectuales, sino que deben ser tomadas como problemas reales insertos en la vida de individuos particulares, en la actividad de comunidades científicas o en la dinámica de grupos sociales. Con ello, mi enfoque se desvía desde el principio de uno muy popular en la epistemología contemporánea que, siguiendo a Wittgenstein (1969), tiende a considerar toda empresa de fundamentación del conocimiento como un absurdo filosófico completamente desconectado de los intereses y conflictos de nuestra vida.

\section{El punto de vista de la teoría de la argumentación}

En este trabajo seguiré una vía que recientemente han explorado Martin Davies (2004 y 2006) y, de manera independiente, Douglas N. Walton (2006), si bien mis planteamientos y resultados difieren considerablemente de los de ambos. Siguiendo esta vía, examinaré el problema de la circularidad en la fundamentación del conocimiento a partir de los resultados ya existentes en el ámbito de la teoría de la argumentación sobre argumentos circulares. Aunque a primera vista los argumentos tienen finalidades distintas (en el caso argumentativo, convencer con buenas razones al oyente; en el caso epistémico, producir nuevos conocimientos) y distintos criterios de corrección, lo cierto es que ambos casos comparten entre sí una importante propiedad: la introducción de consideraciones pragmáticas y contextuales, que no son tenidas en cuenta en el enfoque lógico.

Podemos caracterizar esquemáticamente el contexto en que aparece un argumento a través de los participantes y la serie de proposiciones que ellos profieren o que intervienen en la argumentación de otra manera. Así, un contexto argumental está constituido por un conjunto de preguntas (enunciados en torno a cuyo valor de verdad se argumenta) una serie de participantes, cada uno con una serie de compromisos (enunciados cuya verdad acepta); un conjunto de 
supuestos, incluyendo tanto supuestos de fondo (enunciados que todos los participantes aceptan al principio de la argumentación) como supuestos que se han ido abriendo a lo largo de la argumentación y un historial de movimientos previos, ya sea la formulación de argumentos o la introducción de nuevos compromisos, preguntas o supuestos. Un contexto epistémico está conformado por un conjunto de objetivos (enunciados cuyo valor de verdad desea ser conocido), uno o varios sujetos epistémicos cada uno con un conjunto de creencias, un conjunto de supuestos tanto de fondo como introducidos en el curso de la argumentación, y un historial de movimientos previos, incluyendo el recorrido de argumentos y la introducción de evidencias y supuestos nuevos. En ambos casos, además, existen una serie de reglas (argumentales o epistémicas, según el caso) que determinan qué movimientos son lícitos en cada momento y cuáles son las consecuencias de los mismos respecto a los conjuntos de objetivos, supuestos y compromisos o creencias. En particular, tales reglas determinan cuando la adopción de un compromiso, la apertura de un supuesto, o, en el caso epistémico, una creencia, son lícitos o no. Utilizaré aquí el término general de "aceptar $p$ " para todas estas actitudes proposicionales, y el término "respaldo" para referirme a los razones que hacen de la aceptación de $p$ algo lícito. En el caso especial de la creencia, seguiré la convención habitual de denominar a tal respaldo justificación.

Como he dicho al comienzo de este apartado, mi intención es examinar un asunto epistemológico desde la teoría de la argumentación, así que, por razones puramente metodológicas (ya que deseamos hablar de falacias), entenderé a partir de ahora los contextos epistémicos como una clase de contextos argumentales, para lo cual basta que tomemos los objetivos como una clase de preguntas, las creencias como una clase de compromisos, la introducción de evidencias como una clase de introducción de nuevos compromisos, y las reglas epistémicas como una clase de reglas argumentales. Para el caso en que haya sólo un sujeto epistémico basta con entender que en todos los argumentos el proponente y el receptor son el mismo participante. $^{2}$

${ }^{2}$ Una comparación atenta de ambos tipos de contextos tendría un indudable interés teórico, pero seguramente acabaría alejándonos de nuestro camino. En mi opinión, en muchos casos no vale la pena distinguir entre ambos contextos; por ejemplo, en una argumentación en la que el principal objetivo sea cognoscitivo y los participantes compartan los datos de partidos, el contexto argumental es, directamente, un contexto epistémico. En algunos casos de contextos epistémicos tal vez resulte forzado hablar de contexto argumentativo: por ejemplo, cuando un individuo se forma creencias perceptivas sin que medien (o parezcan mediar) inferencias. 
La manera más habitual de obtener un respaldo para aceptar un enunciado es introduciendo un argumento. En este caso, las premisas del argumento son el respaldo de la conclusión. La propiedad más interesante de un argumento en un contexto argumentativo es muy similar a la propiedad estrella desde el punto de vista epistémico, y por ello utilizaré la misma palabra en ambos casos: convincente. Un argumento es convincente en un contexto argumental si fuerza al participante al que va dirigido a comprometerse o aumentar el compromiso con la conclusión. Un argumento es convincente en un contexto epistémico si proporciona al sujeto epistémico que lo sigue la justificación para creer la conclusión, o aumenta la justificación para creer la conclusión. De otra manera: un argumento es convincente si las premisas son un respaldo para la aceptación de la conclusión en el contexto en que se introduce el argumento. Que un argumento sea convincente o no en un determinado contexto depende de las reglas argumentales o epistémicas. Cuando un argumento que no es convincente conduce a un participante en una argumentación a adoptar (no forzadamente) el compromiso con la conclusión, o a un sujeto epistémico a creer (no justificadamente) la conclusión, decimos que se ha producido una falacia. ${ }^{3}$

Mi propósito con respecto a argumentos fundamentadores como MOORE es determinar si son convincentes, y si no es así, cuál o cuáles falacias de las distintos tipos que se discuten en la teoría de la argumentación tiene la culpa de ello. Como veremos, algunos autores (entre los que me encuentro) defienden que un argumento

Igualmente, en algunos casos de contextos argumentativos como los de la clásica disputatio, en que el principal objetivo es persuasivo, la dimensión epistémica aparece más difuminadamente. En los casos que tratamos en este artículo, en los que uno o varios sujetos intentan averiguar si saben o no saben determinados enunciados, pienso que no sólo conviven ambas dimensiones epistémica y argumentativa, sino que prácticamente se identifican.

3 Nota bene: digo "forzar" y no "incitar" o "impulsar" porque el hecho de que el oyente deba o no comprometerse con la conclusión depende, como venimos diciendo, de reglas constitutivas de las prácticas argumentativas, y no del capricho o la buena voluntad del oyente. Tal y como yo lo entiendo, la propiedad de ser convincente posee un carácter normativo y objetivo (aunque relativo al contexto) de la que carece la propiedad más débil de ser persuasivo, de marcado carácter psicológico. En ocasiones se utilizan en castellano términos como "conclusivo", "correcto" o simplemente "bueno" para referirse a esta propiedad, mientras que en inglés es muy habitual utilizar el término "cogent". Nótese también que hablo de "aumentar el compromiso con la conclusión" porque, para que un argumento sea convincente, no es imprescindible que zanje definitivamente la cuestión. No incluir este elemento "gradual" dejaría fuera no sólo los argumentos inductivos que discutimos aquí, sino también muchos argumentos deductivos (sobre esto volveré en la sección 8). 
circular puede ser convincente en algunos contextos argumentales, así que es necesario que comencemos dando una definición neutral del fenómeno de la circularidad.

Definición de Circularidad. Un argumento A es circular en un contexto argumentativo CA cuando se ha hecho uso de la conclusión $\mathrm{C}$ de A en el contexto CA. Se hace uso de C en un contexto CA si:

(a) C es una de las premisas del argumento A, o se sigue directamente de una o más premisas de A (circularidad de premisas);

(b) C es uno de los supuestos del contexto CA, o se sigue directamente de uno o más de los supuestos de CA (circularidad de supuestos);

(c) C es una premisa de uno o varios argumentos que aparecen anteriormente a A en el historial de CA, o se sigue directamente de una o más premisas que aparecen anteriormente a $\mathrm{A}$ en el historial de CA (circularidad cíclica);

(d) $\mathrm{C}$ es la descripción de una regla de inferencia que ha sido utilizada en algún argumento previo a A en el historial de CA (circularidad de reglas).

MOORE ejemplifica la circularidad de supuestos. El argumento (B) en la secuencia de argumentos siguiente, que se basa en la que aparece en Walton 1994, es un ejemplo de circularidad cíclica:

ARGUMENTO ECONOMÍA

(A) $\left(\mathrm{E}_{1}\right)$ Las empresas abandonan el país.

$\left(\mathrm{E}_{2}\right)$ Si las empresas abandonan el país, la economía anda mal.

$\left(\mathrm{E}_{3}\right)$ Por lo tanto, la economía anda mal.

(B) $\left(\mathrm{E}_{3}\right)$ La economía anda mal.

$\left(\mathrm{E}_{4}\right)$ Si la economía anda mal, las empresas abandonan el país.

( $\left.\mathrm{E}_{5}\right)$ Por lo tanto, las empresas abandonan el país. 
Un buen ejemplo de circularidad de reglas, muy discutido últimamente, es el argumento que propone Boghossian 1996 para justificar la regla de modus ponens utilizando el mismo modus ponens.

\section{ARGUMENTO BOGHOSSIAN}

$\left(\mathrm{B}_{1}\right) \mathrm{Si}$ “ $\rightarrow$ ” significa lo que significa, entonces el modus ponens (MP) es válido.

$\left(\mathrm{B}_{2}\right)$ “ $\longrightarrow$ ” significa lo que significa.

ergo, $\left(\mathrm{B}_{3}\right)$ El modus ponens es válido.

Para el caso (a) vale cualquier argumento del tipo "A, por lo tanto A". Aprovecharé para hacer una observación. Como se desprende de los ejemplos proporcionados, la circularidad no es un fenómeno asociado sólo al conocimiento de naturaleza perceptiva. También se hace presente en ámbitos del conocimiento a priori, como el matemático, el lógico, o el autoconocimiento. Si bien hay suficientes similaridades entre los distintos casos como para poder hablar de un fenómeno general común, existen algunas peculiaridades que harían el tratamiento simultáneo excesivamente prolijo, por lo que en lo que resta de trabajo me centraré en los argumentos fundamentadores asociados al conocimiento perceptivo, dejando para un formato más extenso la más ambiciosa empresa general. ${ }^{4}$

\section{Petición de principio}

Vayamos ahora con la consideración de las falacias que se pueden estar cometiendo en MOORE y argumentos afines. El candidato más obvio es, por supuesto, la falacia de petición de principio. Hablando informalmente, alguien comete esta falacia cuando, intentando probar un enunciado $\mathrm{C}$, introduce indebidamente en el curso de la argumentación a $\mathrm{C}$, con lo cual termina concluyendo $\mathrm{C}$ sólo por el hecho de que ya había partido de él. Supongamos que Carlos me dice que lo que dice Juan ahora es verdad, y que sabe tal cosa porque Juan siempre dice la verdad, pero cuando le pregunto como sabe que Juan siempre dice la verdad él me responde diciendo que Juan se lo

\footnotetext{
${ }^{4}$ En Vilanova 2007 se examina el problema de la circularidad en el conocimiento lógico, y en Vilanova 2009 discuto problemas asociados a la justificación del conocimiento introspectivo.
} 
ha dicho. ${ }^{5}$ Parece obvio que, en este caso, Carlos puede concluir que Juan siempre dice la verdad simplemente porque está suponiendo tal cosa. Si alguien, por ejemplo Pedro, piensa que Juan es un redomado embustero, entonces el argumento de Carlos no servirá para alejarle ni un ápice de su creencia. Esto nos sirve para obtener la clave de la petición de principio: si alguien duda de la conclusión, entonces no puede aceptar las premisas como respaldo de la conclusión. Adviértase que esto no ocurre con todos los argumentos: normalmente, si pensamos que el argumento es válido (deductiva o inductivamente) y dudamos de la conclusión, tenemos buenas razones para dudar de las premisas, pero seguimos teniendo buenas razones para pensar que si las premisas fueran verdaderas la probabilidad (o la plausibilidad) de que la conclusión fuera verdadera aumentaría. Si dudo que Juan sea una buena persona, dudaré que ayude al prójimo, pero seguiré pensando que sería una buena razón para pensar que Juan es una buena persona el contemplar cómo ayuda al prójimo. Sin embargo, si dudo que Juan sea sincero, no sólo dudare que me dice la verdad cuando me dice que él es sincero, sino que además no podré seguir pensando que es una buena razón para pensar que Juan es sincero el hecho de que Juan me lo ha dicho. En la mayoría de los casos, la duda de la conclusión lleva a la duda sobre las premisas; en el caso de la petición de principio, la duda de la conclusión lleva a la duda sobre la misma relación entre premisas y conclusión. Por eso el argumento que comete petición de principio no es convincente: uno no puede verse forzado a aceptar la conclusión ya que si duda de la conclusión duda de que las premisas le fuercen a aceptar la conclusión. Además, éste es un fenómeno exclusivo de la falacia de petición de principio, que no se da en otras falacias, así que hemos llegado a una primera definición general de la petición de principio:

Definición de Petición de Principio 1. En un argumento A en un contexto argumental CA hay petición de principio cuando una duda sobre su conclusión $\mathrm{C}$ produce inmediatamente que las premisas $\mathrm{PP}$ no puedan ser aceptadas como un respaldo para $\mathrm{C}$ en el contexto CA.

En la literatura sobre petición de principio uno se encuentra definiciones más específicas (y más largas). Ello se debe al hecho de que el autor tiene en mente un tipo de circularidad concreta: de premisas, de supuestos, de reglas, o cíclica. Lo cierto es que especificar

${ }^{5}$ El ejemplo es menos nimio de lo que puede parecer a simple vista, pues de hecho es análogo a un muy debatido postulado de las religiones de la revelación. Así, para muchos teólogos cristianos la mayor evidencia de que la Biblia es palabra de Dios es precisamente que en la Biblia se dice que la Biblia es palabra de Dios. 
la definición para un tipo de especialidad tiene sentido, porque los requisitos que han de darse para que se produzca petición de principio pueden variar de un tipo a otro. Sin ir más lejos, hay buenas razones para pensar que la circularidad de reglas es un caso muy especial, pues la duda sobre la conclusión no interfiere con una actitud proposicional (creer una premisa, adoptar un supuesto), sino con un saber cómo (saber cómo hacer un tipo de inferencias, por ejemplo). Pero es importante no perder de vista el fenómeno general. Primero, porque de la comparación entre los distintos tipos de circularidad podemos extraer enseñanzas importantes, como se verá en apartados siguientes. Y segundo, porque en las argumentaciones reales muchas veces no está claro el lugar que ocupa una proposición, por lo que tal distinción sólo aparece en el análisis teórico. Sin ir más lejos, en el ejemplo recién citado tendríamos dificultades para elegir entre las siguientes esquematizaciones:

ARGUMENTO JUAN 1 (circularidad de premisas)

(1) Juan dice que todo lo que dice Juan es verdadero.

(2) Todo lo que dice Juan es verdadero.

(3) Por lo tanto, todo lo que dice Juan es verdadero.

ARGUMENTO JUAN 2 (circularidad cíclica)

(A)

(1) Todo lo que dice Juan es verdadero.

(2) Por lo tanto, si algo es dicho ahora por Juan es verdadero.

(B)

(2) Si algo es dicho ahora por Juan, es verdadero.

(3) Lo que dice Juan ahora es que todo lo que dice Juan ahora es verdadero.

(4) Por lo tanto, todo lo que dice Juan es verdadero. 
ARGUMENTO JUAN 3 (circularidad de supuestos)

(1) Lo que dice Juan ahora es verdadero.

(2) Lo que dice Juan ahora es que todo lo que dice Juan es verdadero.

(3) Por lo tanto, todo lo que dice Juan es verdadero.

Donde el respaldo para aceptar (1) es que he oído a Juan decir (1), bajo el supuesto de que todo lo que dice Juan es verdadero.

ARGUMENTO JUAN 4 (circularidad de reglas)

(1) Juan dice que todo lo que dice Juan es verdadero.

(2) Por lo tanto, todo lo que dice Juan es verdadero.

Donde (2) se infiere de (1) mediante la siguiente regla de inferencia: "De 'Juan ha dicho $p$ ' infiere ' $p$ '."

En cualquier caso, como he dicho, es conveniente disponer de definiciones más específicas. Comenzaré dando, de manera tentativa, el siguiente paquete de definiciones, que pretenden recoger las caracterizaciones más clásicas de la petición de principio, ${ }^{6}$ y que, ya lo advierto, en apartados posteriores revisaremos críticamente:

Definición de petición de principio 1.1. Un argumento A circular de premisas comete la falacia de petición de principio en un contexto argumental CA si la duda de la conclusión C produce de manera inmediata que no se pueda aceptar una premisa o varias de las premisas PP de A en el contexto CA.

Definición de petición de principio 1.2. Un argumento A circular cíclico comete la falacia de petición de principio en un contexto argumental CA si la duda de la conclusión C produce que no se puedan aceptar uno o varios argumentos que han aparecido en el

${ }^{6}$ Así, la definición 1.1 se basa en la de Copi 1961, de la que se puede encontrar una versión refinada en Davies 2006. La definición 1.2 se basa en la que proporciona Jackson 1987. La definición 1.3 se basa en la de Hamblin 1971. La definición 1.4 recoge una caracterización provisional de Boghossian 1996 del tipo de circularidad presente en la fundamentación del conocimiento lógico. 
contexto argumental CA, y el rechazo de tales argumentos produce que no se pueda aceptar el respaldo para una o varias de las premisas $\mathrm{PP}$ de $\mathrm{A}$ en el contexto CA.

Definición de petición de principio 1.3. Un argumento A circular de supuestos comete la falacia de petición de principio en un contexto argumental CA si la duda de la conclusión C produce que no se puede aceptar un supuesto de CA, y el rechazo de tal supuesto produce que no se puede aceptar el respaldo para una o varias de las premisas PP de A en el contexto CA.

Definición de petición de principio 1.4. Un argumento A circular de reglas comete la falacia de petición de principio en un contexto argumental CA si la duda de la conclusión C produce que no se pueda aceptar una regla de inferencia que es necesaria para derivar $\mathrm{C}$ de las premisas PP de A.

Adviértase que las definiciones 1.2 y 1.3 tienen un rasgo en común, rasgo que no poseen las restantes. $\mathrm{Y}$ es que, en ambos casos, la duda de la conclusión produce el rechazo del respaldo para una o varias de las premisas. En el primer caso ello se debe a que tenemos que revisar argumentos que han aparecido anteriormente en la argumentación. En el segundo caso se debe a que hemos de revisar algunos de nuestros supuestos. Pero en los dos la duda sobre la conclusión afecta a la relación entre el respaldo de las premisas y las premisas. Debido a esta afinidad, en este trabajo me centraré en estos dos tipos de circularidad. La circularidad de premisas podemos dejarla fuera por ser muy grosera, y la circularidad de reglas requiere un tratamiento especial que nos tomaría demasiado tiempo y nos desviaría de nuestro objetivo principal. ${ }^{7}$

\section{Apelación a la ignorancia}

En la literatura reciente sobre la circularidad en la fundamentación del conocimiento siempre se hace referencia a la falacia de petición de principio. Curiosamente, casi nunca se menciona la falacia de apellatio ad ignorantiam, a pesar de que ésta ocupa un lugar central en el reto planteado por el escéptico a los sistemas epistémicos o a las fuentes de evidencias.

Intentaré mostrar cómo se llega a esta nueva acusación. Una vez que el escéptico (Sexto Empírico, Hume, Kripkenstein o cualquier

\footnotetext{
${ }^{7}$ Véase Vilanova 2007 para una discusión de la circularidad de reglas y el argumento de Boghossian.
} 
otro) denuncia la circularidad presente en argumentos fundamentadores, no se contenta con obtener la consecuencia de que nunca podremos llegar a saber la conclusión del argumento, sino que deriva un resultado mucho más inquietante: que de hecho nunca hemos sabido las premisas. En el caso de MOORE, la queja del escéptico es que, teniendo en cuenta que podemos derivar el hecho de que nuestro sistema perceptivo funciona bien desde nuestras creencias de origen perceptivo, entonces deberíamos saber que nuestro sistema perceptivo funciona bien antes de empezar a obtener nuestras creencias perceptivas. Dicho en términos que agradarían a un profesor de Lógica: si saber las premisas es una causa suficiente para saber la conclusión, entonces saber la conclusión es una causa necesaria para saber las premisas. Esto demuestra, según el escéptico, que el movimiento ilícito se había dado ya al principio, cuando empezamos a formarnos creencias en torno a lo que ocurría en el mundo externo a partir del testimonio de nuestros sentidos, sin habernos asegurado previamente de que nuestros sentidos funcionaban correctamente. La única defensa que podemos alegar ante el escéptico es que, cuando comenzamos nuestro proyecto epistémico (en el caso de Moore, cuando empezó a acumular evidencias perceptivas), no disponíamos de elementos de juicio para llevar a cabo la tarea de fundamentar nuestras reglas epistémicas. Es decir, no es que tuviéramos evidencias a favor de la fiabilidad de nuestros sentidos, sino que no teníamos evidencias en contra: no había razones para dudar de ellos. Dicho más coloquialmente, dado que no teníamos ninguna evidencia de que algo iba mal, simplemente actuamos como si todo fuera bien. Por muy honesto que este último movimiento sea por nuestra parte, plantea una nuevo problema, y es que el argumento recién invocado (que supusimos que nuestros sentidos nos daban información fiable porque no teníamos razones en contra) recuerda demasiado la falacia de apelación a la ignorancia.

La falacia de la apelación a la ignorancia es mucho más simple que la petición de principio, así que no tomará mucho tiempo caracterizarla. En esencia, se produce cuando inferimos indebidamente $p$ del hecho de que no sabemos no $p$, o, en el caso más sofisticado, del hecho de que no poseemos evidencias en contra de $p$. Es una falacia especialmente peligrosa, pues puede llegar a persuadir de las ideas más excéntricas o levantar los cargos más insospechados. Así, alguien puede concluir que existen duendes invisibles que habitan en los ultramarinos del hecho de que nadie tiene ninguna prueba en contra, o alguien puede concluir que Juan es un redomado hipócrita 
que oculta sus auténticos sentimientos a todo el mundo del hecho, una vez más, de que nadie puede probar lo contrario.

De acuerdo con este análisis provisional, que recoge el tratamiento clásico, ${ }^{8}$ llegamos a la siguiente definición:

Definición de Apelación a la ignorancia 1. Un argumento A en un contexto argumental CA comete la falacia de apelación a la ignorancia cuando tiene la forma:

(1) No hay evidencias en contra de que $p$.

(2) Por lo tanto, $p$.

Es cierto que en el caso epistémico casi nunca uno ha seguido explícita y conscientemente el argumento de la definición de apelación a la ignorancia 1 , sino que se confía en la fuente de evidencias de una manera natural, espontánea. Pero no creo que estemos forzando las cosas si decimos que el argumento se está siguiendo inconscientemente en el momento en que se confía espontáneamente en la fuente de evidencias o, cuando menos, que dicho argumento aparece en la reconstrucción que el propio sujeto epistémico efectuaría a la hora de acometer la tarea de fundamentación de su conocimiento. ${ }^{9}$ En el caso tanto de MOORE como de COHEN hemos de incluir el siguiente argumento en la reconstrucción teórica:

(1) No tengo evidencias en contra de que mis sentidos funcionen defectuosamente.

(2) Por lo tanto, tengo un respaldo para aceptar el supuesto de que mis sentidos funcionan correctamente.

Ahora que ya hemos hecho un primer análisis - aunque provisional- de las falacias de petición de principio y apelación a la ignorancia, es el momento de aplicarlo a los argumentos fundamentadores.

${ }^{8}$ Por ejemplo, Copi 1961.

${ }^{9}$ Para autores como Alston 1986 o Davies 2004 la cuestión de si el supuesto es o no consciente sí es importante. En el caso de Davies, incluso determina que haya petición de principio (paradójicamente, si el supuesto es consciente). En Vilanova 2008 discuto esta postura (así como la postura más radical de Prior 2004, para quien el supuesto no es necesario ni siquiera tomado inconscientemente), discusión en la que no entro aquí porque, como he dicho, es suficiente considerar que el supuesto se hace consciente al menos en el momento en que se inicia la tarea de fundamentación. 
¿Son nuestros dos ejemplos de argumentos fundamentadores doblemente falaces? En el caso de COHEN es clarísimo que el argumento es culpable de ambos cargos. Si alguien duda de la conclusión, es decir, si duda que sus sentidos estén funcionando correctamente en el momento en que percibe el objeto rojo encima de la mesa, entonces no podrá seguir aceptando su experiencia perceptiva como una buena justificación para creer la premisa 1. Además, teniendo en cuenta que la única justificación que se había dado para tomar como supuesto que sus sentidos funcionaban correctamente era que no había evidencia en contra, el argumento claramente incurre en apelación a la ignorancia.

¿Qué ocurre con MOORE? Lamentablemente, de acuerdo con las definiciones que hemos proporcionado el argumento es también doblemente falaz. Por un lado, G.E. Moore no proporciona razones para adoptar el supuesto de que sus sentidos son fiables. En segundo lugar, si eliminamos ese supuesto desaparece el respaldo para cada premisa de la forma "Es el caso que $p_{n}$ ". Y sin embargo, uno no puede dejar de pensar que, si bien COHEN es un argumento perfectamente inútil para fundamentar nuestro conocimiento perceptivo, MOORE ofrece algún tipo de evidencia (por muy pequeña que ésta sea) a favor de que nuestros sentidos no funcionan mal. Efectivamente, es de sentido común que el hecho de que nuestras experiencias perceptivas concuerden entre sí y se confirmen unas a otras es, cuando menos, una buena razón para pensar que todo va bien. De hecho, en los casos mencionados de personas con desórdenes perceptivos o psicológicos tal concordancia no se da, y precisamente eso hace factible que estas personas lleguen a darse cuenta de sus problemas. Que la indagación pueda llegar a un resultado negativo es una prueba de que no hemos hecho trampa. Es decir, que Moore no obtiene el resultado de que sus sentidos son fiables simplemente porque lo supuso al principio, sino que hay algo más que le permite inferir $\left(\mathrm{M}_{3}\right)$. Ese algo más es precisamente lo que falta en el caso en el que se llega a una respuesta negativa.

Si se está de acuerdo con la consideración previa, entonces nuestro siguiente paso es detectar qué errores cometimos en nuestras definiciones de ambas falacias, y proceder a su reformulación.

\section{Legitimación}

Comencemos con la apelación a la ignorancia. Para empezar, hay algunos contextos epistémicos en los que la ausencia de evidencias en contra de $p$ es una buena justificación para creer que $p$. Esto ocurre 
cuando tenemos alguna justificación para creer que, de ser $p$ falso, deberíamos tener evidencias de ello. Si, por ejemplo, efectuamos una minuciosa investigación para comprobar que Pedro no roba material de oficina de su lugar de trabajo y obtenemos resultados negativos, podemos concluir justificadamente que Pedro no roba material de oficina. La estructura del argumento es ahora distinta:

\section{AD IGNORANTIAM 2}

(1) No hay evidencias en contra de que $p$.

(2) Si $p$ fuera falso, deberíamos poseer alguna evidencia en contra de que $p$.

(3) Por lo tanto, $p$.

No parece, sin embargo, que este argumento pueda resolver por sí solo el problema en el caso epistémico. El supuesto de que la fuente de evidencias es fiable funciona desde el principio del proyecto epistémico. En el caso de MOORE, desde el primer momento en que Moore comenzó a adquirir creencias de origen perceptivo. Y en ese momento no sólo no hay evidencias ni a favor ni en contra del supuesto, sino tampoco evidencias a favor de (1). De hecho, no se dispone de tales evidencias hasta que se comienza la tarea de fundamentación del conocimiento y se formula el argumento MOORE. Así que dejaré a un lado, de momento, el argumento 2 (sobre el que volveré más adelante).

Afortunadamente, el argumento 2 no es la única posibilidad. Hay que tener en cuenta que la noción de respaldo y la noción emparentada de aceptación son muy generales, y que admiten distintas variantes cada una con sus propias cualificaciones. En concreto, el tipo de respaldo para adoptar un supuesto es más fácil de obtener que el tipo de respaldo para la creencia. En el caso de la creencia, el respaldo, la justificación, está ligado a la verdad de la proposición creída: si un sujeto epistémico $S$ posee una justificación para creer una proposición $p$, entonces $S$ debe tener razones para creer que es más probable que $p$ sea verdadera que si no tuviera tal justificación. Para el caso de la adopción de un supuesto, sin embargo, basta con poseer alguna razón de índole pragmática que aconseja la adopción del supuesto de que $p$. Walton 1999 da un magnífico ejemplo al respecto: si uno no tiene pruebas a favor de que el arma está descargada, 
es conveniente adoptar el supuesto de que el arma está cargada mientras la manipula. En este caso, la estructura del argumento obedece al siguiente esquema:

\section{AD IGNORANTIAM 3}

(1) No hay evidencias en contra de que $p$.

(2) Hay razones pragmáticas para adoptar el supuesto de que $p$.

(3) Por lo tanto, suponemos que $p$.

El tipo de razones pragmáticas a tener en cuenta puede variar mucho de un contexto argumental a otro, aunque en general tendrán mucho que ver con el objetivo de la argumentación (en el ejemplo de Walton es de suponer que el objetivo es decidir un curso de acción relacionado con el arma). Además, puede haber también algún tipo de justificación teórica a favor de que las razones pragmáticas aducidas son las adecuadas (evidentemente, en el caso del arma hay algunas consideraciones teóricas de fondo sobre el funcionamiento de las armas y las consecuencias físicas de un disparo). Por otro lado, no hay ningún impedimento para que combinemos 2 y 3 y producir un argumento mucho más fuerte:

\section{AD IGNORANTIAM 4}

(1) No hay evidencias en contra de que $p$.

(2) Si $p$ fuera falso, deberíamos poseer alguna evidencia en contra de que $p$.

(3) Hay razones pragmáticas para adoptar el supuesto de que $p$.

(4) Por lo tanto, $p$.

Utilizaré, para ese tipo de respaldo más débil que la justificación, el nombre de "legitimación". ${ }^{10}$ Como ya indiqué antes, la única

${ }^{10}$ Tomo el término en el sentido en que lo usa Wright 2004, donde se explica con detalle tanto la noción como la estrategia para resolver el problema de la fundamentación del conocimiento a partir de ella. Me apresuraré a advertir que mi solución global será muy distinta, y opuesta, a la de Wright, quien opina que los argumentos fundamentadores son todos falaces. Véase Vilanova 2008 para un examen crítico de la postura de Wright. 
diferencia entre la justificación y la legitimación es la relación que guarda con el hecho expresado por $p$. La legitimación no requiere evidencias o indicios de que la proposición legitimada es verdadera. Si $X$ es una justificación para $p$, que yo posea $X$ hace a $p$ más probable (o verosímil) para mí que si no poseyera $X$. En cambio, si $X$ es una legitimación para $p$, que yo posea $X$ no hace más probable (o verosímil) p que si yo no poseo $X$. Esta diferencia determina cuáles son las actitudes epistémicas que pueden respaldar. La justificación puede servir de respaldo para una creencia o un conocimiento. La legitimación sólo puede servir de respaldo para actitudes epistémicas más débiles: la adopción de un supuesto, el compromiso, o el actuar como si $p$ fuera verdadero.

En el caso de un proyecto epistémico, el objetivo es siempre obtener conocimiento, así que uno estará legitimado para suponer que $p$ si tiene razones para pensar que esto ayudará a llevar a buen puerto su proyecto epistémico. Desde luego, no cualquier supuesto vale. No valdrán, por ejemplo, supuestos para los que haya evidencias en contra, o supuestos demasiado excéntricos. Por ejemplo, si hubiera un oráculo divino que me comunicara telepáticamente el tiempo que va a hacer mañana cada vez que cierro los ojos y dejo mi mente en blanco, no cabe duda de que constituiría una vía inmejorable para hacer predicciones metereológicas, pero está claro que no tengo legitimidad alguna para hacer tal supuesto. Hay otras consideraciones que se podrían tener en cuenta aquí. Por ejemplo, si es una buena razón para suponer que $p$ el hecho de que sólo bajo el supuesto de que $p$ puedo tener alguna posibilidad de obtener conocimientos. $\mathrm{O}$ si razones de índole meramente material, como la capacidad de procesamiento, los límites de espacio y tiempo e incluso el presupuesto económico del que se dispone pueden ser tenidos en cuenta. Pero, para no abundar demasiado, basta con entender que en un proyecto epistémico uno está legitimado a adoptar $p$ como supuesto si tiene razones para pensar que es más probable que obtenga mejores y más conocimientos si supone $p$ que si no supone $p$.

Además, razones de ese tipo se pueden obtener mediante algún cálculo a priori, o comparando proyectos epistémicos previos (si es que los hay), así que en muchos casos (incluyendo los casos que nos interesan) el esquema de argumento ad ignorantiam 3 puede ser seguido, y obtener una legitimación para hacer la suposición antes de que el proyecto epistémico sea puesto en marcha. En el caso de MOORE, uno puede mediante pura reflexión llegar a la conclusión de que tiene más probabilidades de obtener conocimientos sobre el mundo externo haciendo el supuesto de que sus sentidos son fiables 
que adoptando cualquier otro supuesto que se le ocurra, incluyendo oráculos divinos y similares. Tenemos así un nuevo contexto argumental para MOORE, en el que se ha propuesto el argumento que sigue el esquema ad ignorantiam 3 , y en el que no se comete la falacia de apelación a la ignorancia.

\section{Petición de principio reformulada}

Vayamos ahora con la petición de principio. Sin salirse del ámbito de la teoría de la argumentación, hay ya varias razones para pensar que tanto la definición 1.3, que es la que aquí interesa, como la definición emparentada 1.2, son demasiado restringidas. ${ }^{11}$

Para empezar (y aunque este punto no es especialmente relevante aquí) incluso en los casos más flagrantes de petición de principio los argumentos pueden resultar útiles en el curso de una argumentación. Obviamente no pueden servir para convencer a nadie de su conclusión, pero sí pueden servir para que quien ya está convencido previamente de su conclusión se percate de una relación lógica entre sus compromisos. ${ }^{12}$ Este dato nos pone sobre aviso con respecto al hecho de que hay que tomar en consideración todos los aspectos del contexto de argumentación en un sentido amplio, tales como el objetivo del argumento. No siempre es el de demostrar definitivamente que la conclusión es verdadera. Puede haber otros objetivos, como explicitar una relación lógica (de esto se sigue necesariamente esto, esto es contradictorio con esto, esto aumenta la probabilidad de esto, etc.) o, como vimos antes, justificar un supuesto. De hecho, en la mayoría de las situaciones reales, en las que la información disponible es parcial y la disponibilidad de tiempo limitada, casi nunca el objetivo es el de demostrar la conclusión, sino uno más modesto: añadir verosimilitud. Es poco habitual que en un contexto argumental estemos en disposición de probar una proposición mediante un solo argumento. Normalmente acumulamos argumentos que proporcionan

${ }^{11}$ En este apartado sigo especialmente los trabajos de Douglas N. Walton que se citan en la bibliografía. De él tomo prestados sobre todo los ejemplos y la casuística, si bien tanto mi forma de presentar las cosas como la caracterización final de la petición de principio es diferente.

${ }^{12}$ Es por ello que algunos autores consideran que argumentos como estos no son falaces cuando la finalidad es distinta a la de convencer o zanjar la cuestión en torno a la conclusión. Así, Jackson 1987 habla de la función de "resaltar" (teasing out) y Davies 2006 de la función de "decidir que creer" (deciding what to believe) para el caso epistémico. Dado que voy a defender la tesis más ambiciosa de que se puede evitar la falacia incluso en los casos en que la función del argumento es contribuir a convencer o zanjar la cuestión, no me detendré en este trabajo a examinar dicha vía. 
una verosimilitud creciente hasta llegar a concluir que la proposición en cuestión es considerablemente más verosímil que cualquier otra hipótesis. Cualquier argumento que proporciona mayor verosimilitud a su conclusión, por pequeña que ésta sea, es pertinente en contexto argumentativo, y por lo tanto no es falaz.

En segundo lugar, repárese en que no en todo contexto de argumentación sería rechazada una cadena de argumentos como la de ECONOMíA. En ocasiones se producen en los sistemas económicos estos canales de retroalimentación (peor economía produce más éxodo, que a su vez produce peor economía, que a su vez produce más éxodo...), y pueden aducirse como explicación causal. El círculo argumental no haría aquí más que reflejar el círculo vicioso en la situación real. Recordemos que un argumento no tiene que zanjar definitivamente la cuestión sobre si su conclusión es verdadera para ser convincente; basta con que aumente el grado de verosimilitud en la conclusión. Es posible que, en tanto que explicación causal, ECONOMía no sea suficiente (falta, por ejemplo, dar cuenta del suceso que disparó el círculo vicioso) pero puede ser una buena razón para inclinarnos a pensar que hay un proceso de éxodo empresarial en marcha.

En tercer lugar, adviértase que en una argumentación pueden aparecer o desaparecer dudas, según vayan variando los intereses de los participantes. No hay nada ilícito en que un participante plantee una pregunta que no se había contemplado al principio, siempre y cuando no se desvíe demasiado el tema de conversación y todos los participantes estén de acuerdo. En ocasiones, la nueva duda puede solucionarse simplemente recordando algún resultado previo de la argumentación, lo que provocará un círculo argumental que, sin embargo, no será falaz. Esto se produce en el siguiente ejemplo de historial argumentativo, que es una reescritura de uno proporcionado por Walton 1994:

EJEMPLO DUDA

(A)

(1) Es el caso que $p$.

(2) Si es el caso que $p$, entonces es el caso que $q$.

(3) Es el caso que $q$. 
(B) DUDO que $p$.

(C)

(4) Es el caso que $q$.

(5) Si es el caso que $q$, entonces es el caso que $p$.

(6) Es el caso que $p$.

En este caso, el proponente del argumento (C) estaría haciendo ver a la persona que planteó la duda (B) que rechazar $p$ supone rechazar un compromiso anterior, a saber, el compromiso con $q$, y para ello invoca la nueva evidencia de que $q$ implica $p$ (premisa (5)). Ante esto, el participante que efectuó el movimiento (B) puede hacer varias cosas: quizás se desdiga también de $q$, lo cual puede exigir ulteriores revisiones del historial de la argumentación (entre (A) y (B) $q$ puede haber sido utilizado como premisa de otros argumentos), o quizás opte por aceptar $p$ (tal vez recordando las razones por las que aceptó en su momento la premisa (1)) y eliminar la duda planteada, teniendo en cuenta su compromiso anterior con $q$. Una vez más, el argumento (B) no zanja completamente la cuestión, pero es evidente que introduce un nuevo elemento de juego en la argumentación (una razón para aceptar $p$ ) y no es completamente banal.

Si nos fijamos en lo que tienen en común los dos ejemplos anteriores y que no comparten los ejemplos más claramente falaces como JUAN o COHEN, obtenemos un diagnóstico más certero con respecto a la petición de principio. Al formular los argumentos que comenten la petición de principio no se obtiene ningún respaldo para aceptar la conclusión que sea distinto de aquel del que ya se disponía antes de seguirlo, ya sea que la conclusión fuera un supuesto o un compromiso. Por ello no son convincentes, y por eso resultan peligrosos en la argumentación, porque al seguirlos alguien puede pensar que dispone de una evidencia nueva a favor de la conclusión, y a raíz de ello fortalecer su compromiso con la misma o, en el peor de los casos, dejar de considerarla un supuesto para pasar a tomarla como una proposición probada. Por el contrario, cuando uno sigue un argumento circular convincente obtiene un respaldo diferente para creer la conclusión de que se disponía antes de seguirlo, y ello porque el argumento produce una evidencia nueva que no se había tomado en cuenta cuando la conclusión se aceptó (ya sea como supuesto o como compromiso) antes de seguir el argumento. Que esta evidencia nueva 
sea condicional con respecto a la aceptación de la conclusión como supuesto obviamente disminuye la fuerza probativa del argumento, pero no la reduce a cero. Esto demuestra que las definiciones específicas de petición de principio 1.1 y 1.3 fueron formuladas con demasiada celeridad: se detenían en el hecho de que la duda de la conclusión producía dudas sobre el respaldo a una o más premisas sin tener en cuenta las nuevas consideraciones que introducían las premisas mismas.

Para explicar bien este punto, que es decisivo, quisiera hacer una reflexión global en torno a la estructura y la función de nuestras prácticas argumentativas. Dado que en un argumento siempre entran en juego evidencias y creencias previas, estrictamente hablando el argumento no introduce justificaciones nuevas (como sí lo haría, por ejemplo, un nuevo dato perceptivo), sino que reorganiza las ya disponibles para sacarles más partido y permitirnos justificar una nueva creencia o adoptar un nuevo compromiso (la conclusión del argumento, claro). En general, un argumento nos proporciona información sobre determinadas relaciones entre los respaldos RR que se poseen para cada una de las premisas de PP tomados a la vez. Estas relaciones se expresan de manera indirecta a través de la relación de inferencia entre las premisas y la conclusión. Es decir, la relación entre respaldos que introduce en la discusión o investigación el nuevo argumento consiste en que de ciertas proposiciones respaldadas por RR (las premisas del argumento) se sigue cierta otra proposición (la conclusión). Un argumento es pertinente y útil si, como dijimos antes, las relaciones entre respaldos expresadas por el argumento introducen alguna consideración nueva en la argumentación, que no se tenía en cuenta cuando consideramos aisladamente la relación de cada respaldo con su premisa respaldada. Tomemos ahora un argumento circular cualquiera, A, con premisas PP y conclusión C. Sea PPC el subconjunto del conjunto de las premisas PP del argumento A en cuyo respaldo interviene la conclusión $\mathrm{C}$, ya sea como supuesto de que el respaldo alegado es genuino, ya sea como premisa en un argumento previo. En el caso de los argumentos circulares, comoquiera que la conclusión $\mathrm{C}$ ya forma parte del respaldo de las premisas PPC, se requiere, para que el argumento sea convincente, que la relación entre premisas y conclusión añada algo distinto a la relación entre cada proposición de PPC y su respaldo. Si no es así, hay falacia. Esto puede expresarse de la siguiente manera: la relación entre cada proposición y su respaldo no es la misma que la relación entre las premisas y la conclusión. Llegamos, de este modo, a la siguiente reformulación de las definiciones 1.2 y 1.3 : 
Definición de petición de principio 2.2. Un argumento A circular cíclico con premisas $\mathrm{PP}$ y conclusión $\mathrm{C}$ comete la falacia de petición de principio en un contexto argumental CA si (i) la duda de la conclusión $\mathrm{C}$ produce que no se puedan aceptar uno o varios argumentos que han aparecido en el contexto argumental CA, y (ii) el rechazo de tales argumentos produce que no se pueda aceptar el respaldo RC para una o varias de las premisas $\mathrm{PC}$ de $\mathrm{PP}$, y (iii) para cada una de las premisas $\mathrm{PC}$ y su respaldo correspondiente $\mathrm{RC}$, un participante posee el respaldo $\mathrm{RC}$ para $\mathrm{PC}$ si y sólo si uno posee el respaldo PP para C.

Definición de petición de principio 2.3. Un argumento A circular de supuestos con premisas PP y conclusión C comete la falacia de petición de principio en un contexto argumental CA si (i) la duda de la conclusión $\mathrm{C}$ produce que no se puede aceptar un supuesto de CA, y (ii) el rechazo de tal supuesto produce que no se puede aceptar el respaldo $\mathrm{RC}$ para una o varias de las premisas PC de PP, y (iii) para cada premisa $\mathrm{PC}$ y su respaldo correspondiente $\mathrm{RC}$, un participante tiene el respaldo $\mathrm{RC}$ para $\mathrm{PC}$ si y sólo si tiene el respaldo $\mathrm{PP}$ para $\mathrm{C}$.

Obsérvese que 2.2 y 2.3 no nos obligan a abandonar la definición general de petición de principio l: si dudamos de la conclusión C, según la cláusula (ii) en ambos casos no será posible aceptar el respaldo $\mathrm{RC}$ para las premisas $\mathrm{PC}$, y comoquiera que por la clausura (iii) las premisas son un respaldo para la conclusión sólo si RC son respaldos para $\mathrm{PC}$, entonces las premisas dejan de ser un respaldo para la conclusión. Tenemos, así, un indicio indirecto de que las definiciones 2.2 y 2.3 no son $a d$ hoc, pues caen bajo la caracterización general de la petición de principio.

\section{Argumentos que si fundamentan}

Aplicando las nuevas definiciones al caso epistémico enseguida nos percatamos de que MOORE es convincente y COHEN no lo es. En el caso de COHEN el respaldo $\mathrm{Rl}$ para aceptar la premisa $\left(\mathrm{C}_{1}\right)$ es que la impresión sensorial de que hay un objeto encima de la mesa junto con el supuesto de que el sistema perceptivo funciona bien en este momento. Pero ése es precisamente el presunto respaldo que $\left(\mathrm{C}_{1}\right)$ y $\left(\mathrm{C}_{2}\right)$ ofrecen para $\left(\mathrm{C}_{3}\right)$ : que la impresión sensorial ha producido una creencia verdadera. Así que si uno tiene el respaldo $\mathrm{Rl}$ para $\left(\mathrm{C}_{1}\right)$ automáticamente tiene el respaldo $\left(\mathrm{C}_{1}\right)$ y $\left(\mathrm{C}_{2}\right)$ para $\left(\mathrm{C}_{3}\right)$ : no cabe imaginarse una situación en la que alguien que tuviera el respaldo $\mathrm{R} 1$ no tuviera también las premisas $\left(\mathrm{C}_{1}\right)$ y $\left(\mathrm{C}_{2}\right)$. 
En el caso de MOORE, formulado en el contexto argumental en el que se ha seguido el esquema de argumento ad ignorantiam 3, por el contrario, al tomar todas las premisas a la vez, aparece un respaldo nuevo para la conclusión que es distinto del respaldo que teníamos para aceptar cada una de las premisas $\left(\mathrm{M}_{n}\right.$ bis). En efecto, el respaldo para aceptar la premisa $\left(\mathrm{M}_{1}\right.$ bis) es una determinada experiencia perceptiva $\mathrm{P}_{1}$ bis junto con el hecho de que es pragmáticamente adecuado tomar mis experiencias perceptivas como fiables (conclusión de ad ignorantiam 3). El respaldo para aceptar la conclusión (M) es que cada premisa $\left(\mathrm{M}_{n}\right)$ se ve corroborada por la premisa correspondiente $\left(\mathrm{M}_{n} \mathrm{bis}\right)$. Pero uno puede poseer ese respaldo para alguna de las premisas, por ejemplo $\left(\mathrm{M}_{1}\right.$ bis) y todavía no poseer el respaldo para el resto de premisas. De hecho, podría ser el caso que $\left(\mathrm{M}_{1} \mathrm{bis}\right)$ fuera verdadero, y que (M) fuera falso: mis sentidos podrían haber funcionado bien cuando obtuve la experiencia perceptiva $\mathrm{P}_{1}$ bis y haber funcionado mal en un número considerable de otras ocasiones. Incluso podrían haber funcionado mal en todos los casos restantes que recogen las premisas del argumento MOORE. Así que aquí no se cumple la condición (iii) de la definición de petición de principio 2.3: uno puede poseer el respaldo $\mathrm{RC}$ para una de las premisas $\mathrm{PC}$ y no poseer el respaldo $\mathrm{PP}$ para la conclusión $\mathrm{C}$.

Como es evidente, la defensa que se ha dado para la acusación de apelación a la ignorancia cumple un papel estratégico en el rechazo de la acusación de petición de principio. Pero también se da la recíproca aquí, ya que nuestra respuesta a la acusación de petición de principio también contribuye a la absolución definitiva respecto a la apelación a la ignorancia. En la sección 5 proporcioné un esquema de argumento ad ignorantiam, el esquema 4, que era más fuerte que el esquema de argumento 3 que he venido utilizando hasta ahora. En él, junto a las razones pragmáticas para la adopción de $p$ como supuesto, se añadía la consideración de que si $p$ fuera falso deberíamos poseer alguna evidencia en contra de que $p$. Esto último se daba cuando habíamos llevado a cabo una investigación dirigida a recoger evidencias en contra de que $p$, y esa investigación había dado resultados negativos, algo que no podía ser el caso cuando se suponía $p$ al comienzo del proyecto epistémico. Pero eso sí puede ocurrir en el momento en que se pone en marcha la tarea de fundamentación del conocimiento, cuando el proyecto ya lleva "andando" un buen trecho. Es decir, eso ocurre cuando uno sigue el argumento MOORE y descubre la concordancia entre nuestras experiencias perceptivas: hay razones para pensar que si nuestro sistema perceptivo no funciona correctamente, 
entonces aparecerían contradicciones entre nuestras distintas creencias perceptivas. Es decir, hay razones para pensar que si fuera falso que nuestro sistema perceptivo funciona correctamente, entonces deberíamos tener evidencias de ello. Como no las hay, podemos seguir el esquema ad ignorantiam 4 y concluir $p$ no sólo como supuesto, sino como algo más fuerte: como creencia.

\section{Inferencia y proyecto cognitivo}

Hasta ahora no he dicho nada acerca del tipo de inferencia que tiene lugar en MOORE y otros tipos de argumentos fundamentadores. Aunque he podido llevar esta indagación hasta buen puerto (o así confío) sin tocar ese punto, lo cierto es que tiene relevancia a la hora de determinar el tipo de respaldo cognitivo obtenido para su conclusión. Así que antes de rematar este trabajo, es de justicia echar un vistazo a la misma.

Lo cierto es que Moore no hace más que algunos vagos comentarios sobre el tipo de inferencia involucrado en su argumento, señalando, como he dicho, que puede tratarse de un argumento analógico o inductivo. Desde luego, no se trata de un argumento deductivo. Y resulta muy problemático, igualmente, considerar MOORE como un argumento analógico. Más satisfactoria es la invitación a entenderlo como argumento inductivo: del hecho de que en $n$ casos sus sentidos han tenido éxito en la formación de creencias en torno al mundo externo, Moore concluye que sus sentidos tienen éxito en la formación de creencias en torno al mundo externo. ${ }^{13}$

En todo caso, queda claro que MOORE no es deductivo, y COHEN aparentemente lo es. Esto podría hacer pensar que para que un argumento fundamentador sea cogente es un requisito necesario que no sea deductivo. Y, ciertamente, que un argumento fundamentador sea deductivo concede al escéptico una baza importante en su intento de derribar nuestras pretensiones de conocimiento. Si el argumento es deductivo, entonces es válido si y sólo si es imposible que las premisas sean verdaderas y la conclusión falsa al mismo tiempo. El escéptico proporciona contraejemplos, situaciones en que las premisas serían verdaderas y la conclusión falsa recurriendo a la construcción

\footnotetext{
${ }^{13}$ Una manera más sutil de entenderlo es como un argumento abductivo, del tipo "inferencia a la mejor explicación": la mejor explicación de que nuestras creencias perceptivas concuerden entre sí es que nuestro sistema perceptivo es una fuente fiable de evidencias. De hecho, no hay ningún problema para entenderlo de ambas maneras a la vez o, hablando más rigurosamente, de recorrer dos argumentos, MOORE 1 como inductivo, y MOORE 2 como abductivo.
} 
de escenarios tipo "genio maligno" o "cerebro en la tina". Así que parece que deberíamos descartar los argumentos deductivos.

Sin embargo, existen argumentos fundamentadores que aparentemente siguen las leyes de la lógica clásica de primer orden. BOGHOSSIAN es un buen ejemplo de ello. Otro ejemplo es la secuencia de argumentos que Descartes traza en las Meditaciones metafísicas, cuando intenta probar que la intuición racional es una buena vía de conocimiento a priori recurriendo a resultados obtenidos mediante la intuición. ${ }^{14} \mathrm{O}$ bien, en una aproximación naturalista quizás un tanto extrema, los argumentos que dedujeran la corrección de nuestro sistema perceptivo de teorías científicas aceptadas también se podrían considerar deductivos.

Así pues, ¿qué ocurre con el reto del escéptico? ¿Son inservibles para la fundamentación del conocimiento tales argumentos? Si fuera así, habría que preguntarse qué es lo que les hace no ser convincentes, a pesar de ser deductivamente válidos. En los trabajos recientes sobre esta cuestión se soluciona este problema distinguiendo entre cierre de respaldo (closure of warrant) y transmisión de la justificación (transmission of justification), ${ }^{15} \mathrm{y}$ vinculando sólo el primero a la validez deductiva. Estas soluciones, sin embargo, tienen la consecuencia de que no sólo los argumentos recién citados, sino también MOORE y el resto de argumentos fundamentadores comenten la falacia de petición de principio o, cuando menos, no sirven para aumentar el grado de compromiso con la conclusión. Así que a mí no me sirve, obviamente, esta solución. Afortunadamente, hay una solución a mi entender mucho más simple, y que es consistente con mi planteamiento. Y es que argumentos como BOGHOSSIAN sólo aparentemente siguen las leyes de la lógica clásica. En realidad, la lógica que se está utilizando es una lógica no monótona.

Explicaré esto un poco mejor. Hay cuatro propiedades de la lógica no monótona ${ }^{16}$ que nos hacen pensar que es la utilizada (junto con procedimientos inductivos, abductivos y analógicos) en la tarea de

${ }^{14}$ Véase, por ejemplo, la reconstrucción de Sosa 1997, para quien los argumentos cartesianos no son falaces.

${ }^{15}$ Los términos provienen de Wright 1985, quien a su vez se basa en conceptos de F. Drestke y R. Nozick, y es ampliamente utilizado en Wright 2004, Davies 2004 y Davies 2006 .

${ }^{16}$ Existen varios sistemas de lógica no monótona en el mercado, y no voy a entrar aquí a discutir cuál es la genuina lógica no monótona (aunque cabe pensar que para nuestro caso la más próxima y útil es la lógica autoepistémica), así que hablaré sin más de la lógica no monótona. 
fundamentación del conocimiento, y aclaran algunos aspectos problemáticos de la misma.

Para empezar, las constantes lógicas que utilizan la lógica clásica y la lógica no monótona son las mismas, y también son muy parecidas las reglas de inferencia. Esto hace que, a la hora de formalizar un argumento del lenguaje natural, la cuestión sobre cuál de las dos lógicas utilizar no tenga una respuesta sencilla. No ocurre, pues, lo mismo que con la lógica modal, donde enseguida se advierte la presencia de operadores lógicos de los que carece la lógica clásica ("es necesario que", "posiblemente"...), o con la lógica intuicionista, donde de manera inmediata se observa que hay teoremas de la lógica clásica que no se cumplen. En realidad, sólo cuando se modifican elementos del contexto argumental y eso altera el conjunto de consecuencias de los compromisos (tal y como se explica más adelante) es posible identificar claramente una inferencia no monótona. Esto sirve para explicar por qué argumentos como y similares pueden haber confundido a muchos.

En segundo lugar, una inferencia no monótona válida no garantiza la verdad de la conclusión, sino que tan sólo aporta una plausibilidad relativa: en el mejor de los casos muestra que la conclusión es la más verosímil de las alternativas disponibles. Este hecho concuerda perfectamente con mi análisis de los argumentos fundamentadores, ya que defendí que normalmente uno de ellos no es suficiente para zanjar definitivamente la cuestión sobre la conclusión, sino que simplemente introduce evidencias a favor de la conclusión, que a su vez aumentan el grado de confianza en la misma.

En tercer lugar, al contrario de la lógica clásica, la relación de inferencia no depende sólo de cuáles sean las premisas y cuál la conclusión, sino que introduce un tercer elemento de clara naturaleza cambiante y contextual, el cual recibe distintos nombres según la versión de la lógica no monótona en cuestión ("base de datos" en la lógica de la circunscripción de McCarthy, "descripción de un mundo" en la lógica por defecto de Reiter, o "base de conocimientos" en la lógica autoepistémica). Esta propiedad refleja a la perfección el hecho ya señalado de que el argumento es convincente o no respecto al contexto epistémico.

En cuarto lugar, y éste es el punto más importante aquí, las inferencias no monótonas son inferencias por defecto. Es decir, son inferencias que tienen un carácter provisional, condicionado por las evidencias disponibles y, consecuentemente, pueden ser revisadas ante la aparición de nuevas evidencias. Como ilustra el célebre ejemplo de Tweety. 
Tweety es un pajaro.

Los pajaros vuelan.

Tweety vuela.
Tweety es un pájaro.

Los pájaros vuelan.

pero Tweety es un pingüino.

Tweety no vuela.

La no monotonía no sólo explica que un sistema epistémico para el que en un momento determinado creemos justificadamente que está bien fundamentado, pueda, a medida que avanza el proyecto epistémico y aparecen nuevas evidencias, entrar en crisis y perder la fundamentación. Además, explica el hecho aparentemente paradójico que, como veíamos al comienzo de este apartado, irritaba al escéptico. La propiedad de monotonía de la lógica clásica dice que si de $\mathrm{PP}$ se sigue $\mathrm{C}$, entonces de $\mathrm{PP}$ y $\mathrm{R}$ se sigue $\mathrm{C}$. En las lógicas no monótonas este principio no se cumple siempre, lo que explica por qué si bien de las premisas de un argumento fundamentador convincente se sigue (no monótonamente) la conclusión, de las premisas de dicho argumento fundamentador junto con la hipótesis del escéptico (por ejemplo, el genio maligno) no se sigue (no monótonamente) la conclusión. ${ }^{17}$

\section{REFERENCES}

Alston, W.P., 1986, "Epistemic Circularity", Philosophy and Phenomenological Research, vol. 47, no. 1, pp. 1-30.

Boghossian, P., 1996, "Analyticity Reconsidered", Noûs, vol. 30, no. 3, pp. 360-391.

Boghossian, P. y C. Peacocke (comps.), 2000, New Essays on the A Priori, Clarendon Press, Oxford.

Cohen, S., 2002, "Basic Knowledge and the Problem of Easy Knowledge", Philosophy and Phenomenological Research, vol. 67, pp. 309-329.

Copi, I.M., 1961, Introduction to Logic, Macmillan, Nueva York.

Davies, M., 2006, "Two Purposes of Arguing and Two Epistemic Projects", en I. Ravenscroft (comp.), Minds, Ethics, and Conditionals: Themes

${ }^{17}$ Este trabajo se llevó a cabo al amparo de los proyectos HUM 2006-04955 y FFI 2008-03092 del Ministerio Español de Ciencia e Innovación y el grupo ComplutenseComunidad de Madrid 930174. Versiones preliminares han sido presentadas en el Instituto de Investigaciones Filosóficas de la UNAM y en el Coloquio Compostelano de Filosofía Analítica, y discutidas con miembros de los proyectos. Quisiera agradecer a todos ellos sus muchas sugerencias y ayuda, así como, de una manera especial, a los anónimos árbitros de esta revista y a los profesores Antonio Blanco y Carlos Pereda por su auxilio en la mejora del texto y contenido de la versión final. 
from the Philosophy of Frank Jackson, Oxford University Press, Londres/Nueva York, pp. 337-385.

Davies, M., 2004, "Epistemic Entitlement, Warrant Transmission and Easy Knowledge", Proceedings of the Aristotelian Society, vol. supl. 28, pp. 213-245.

—_ 2000, "Externalism and Armchair Knowledge", en Boghossian y Peacocke 2000, pp. 384-415.

Jackson, F., 1987, Conditionals, Basil Blackwell, Londres.

Moore, G.E., 1940, "Four Forms of Scepticism", en G.E. Moore, 1959, Philosophical Papers, Allen and Unwin, Londres, pp. 196-226.

Pryor, J., 2004, “What's Wrong with Moore's Argument?", Philosophical Issues, vol. 14, pp. 350-378.

Sosa, E., 1997, "How to Resolve the Pyrrhonian Problematic: A Lesson from Descartes", Philosophical Studies, vol. 35, pp. 229-249.

Vilanova, J., 2009, "The Many Faces of Mc Kinsey's Paradox", en Luis Fernández (comp.), Language, Nature and Science, Plaza y Valdés, Madrid, pp. 209-231.

—_ 2008, "Fundamentación sin fundamentalismos", Praxis Filosófica, vol. 27 , pp. 7-31.

— 2007, "Implicit Definitions and Knowledge of Logic", en J.L. Falgueras, C. Martínez y J.M. Sagüillo (comps.), Current Topics in Logic and Analytic Philosophy, Ediciones Universitarias, Santiago de Compostela, pp. 117-138.

Walton, D.N., 2006, "Epistemic and Dialectical Models of Begging the Question", Synthese, vol. 152, pp. 237-254.

—_, 1999, Arguments from Ignorance, Penn State Press, Pensilvania. , 1994, "Begging the Question as a Pragmatic Fallacy", Synthese, vol. 100, pp. 95-131.

- 1991, Begging the Question: Circular Reasoning as a Tactic of Argumentation, Greenwood Press, Nueva York.

Wright, C., 2004, "Warrant for Nothing (and Foundations for Free?)", Proceedings of the Aristotelian Society, vol. supl. 78, pp. 167-212. , 1985, "Facts and Certainty", Proceedings of the British Academy, vol. 31, pp. 429-472.

Recibido el 8 de octubre de 2009; revisado el 9 de junio de 2010; aceptado el 1 de diciembre de 2010. 